\title{
Routine Mask Induction and Intubation without Paralysis may be Safer
}

\author{
Lewis S. Coleman*
}

Department of Anesthesia, Fellow, American Institute of Stress.

"Corresponding author: Lewis S. Coleman, MD, DABA, Department of Anesthesia, Fellow, American Institute of Stress, Tel: 5598023375/(661) 900-2390; E-mail: lewis_coleman@yahoo.com

Citation: Coleman, L.S. Routine Mask Induction and Intubation without Paralysis may be Safer. (2015) J Anesth Surg 2(2): 54-55.

\section{To the Editor}

Meticulous precautions cannot eliminate disasters with presently prevailing induction intravenous techniquesA. Elective mask induction and intubation with Sevoflurane optimizes safety by incorporating pre-oxygenation and de-nitrogenation, eliminating paralysis that unpredictably obstructs the airway, avoiding lethal bolus hypnotic agent toxicity ${ }^{[1]} \mathrm{B}$, and enabling safe retreat from unanticipated anatomical problems by ventilating with pure oxygen. Today the safety of mask induction is largely forgotten, and many practitioners assume that intravenous hypnosis and paralysis are essential to assure patient comfort and prevent dental and vocal cord damage during elective intubation. These assumptions are unfounded ${ }^{[2]}$. Sevoflurane with modern monitoring and synthetic opioids has revolutionized mask induction and intubation without muscle relaxants, and it deserves a fresh look ${ }^{[3]}$.

The dangers and difficulties of rapid intravenous induction, paralysis, and intubation have inspired numerous practitioners to embrace "open airway" techniques with intravenous Propofol infusions, but such techniques sacrifice respiratory assistance, and invite fire, hypoventilation, asphyxiation, aspiration, and airway obstruction that necessitate emergency intubation under difficult and dangerous circumstancesC,D. Like methane and nitrogen, carbon dioxide is an Asphyxiant gas that disrupts oxygen uptake in pulmonary alveoli ${ }^{[3]}$. Pulmonary physiology efficiently expels $\mathrm{CO}_{2}$ in conscious subjects at normal atmospheric levels of $0.03 \%$, but occult $\mathrm{CO}_{2}$ asphyxiation becomes problematic at $\mathrm{FICO}_{2}$ at least as low as $5 \%$, and is lethal above $30 \%$. Capnography cannot measure $\mathrm{CO}_{2}$ in the absence of endotracheal intubation, and open airway pharyngeal gas sampling cannot assess occult alveolar $\mathrm{CO}_{2}$ accumulation that explains the sudden episodes of unexpected oxygen desaturation that plague open airway techniques despite oxygen supplementation.

Surprise and paralysis cause most induction disasters ${ }^{[6]}$. I came to appreciate how fatigue, hunger, and hubris readily paralyze pre-operative preparation as a means to prevent airway disasters after my close encounter with a bearded, overweight, diabetic, middle-aged man who presented as an emergency "add-on" ${ }^{n 7,8]} \mathrm{E}$. Thankfully, the LMA averted disaster. I subsequently embraced routine Sevoflurane mask induction and nasal intubation without paralysis to avoid similar predicaments in outpatient dental clinics where anatomical airway problems were common, and pre-operative assessment was problematic with children and mentally handicapped patients. Risk free induction has yet to be invented, but Sevoflurane mask induction and intubation without paralysis obviates most induction emergencies. It is inherently safer than presently prevailing intravenous induction techniques, and nearly as efficient. Intravenous catheter installation is fast, easy and painless after patients are anesthetized. Mask induction minimizes emergency medication requirements, and epinephrine and atropine can be administered via the endotracheal tube.

After performing routine Sevoflurane mask induction and nasal intubation without paralysis on hundreds of patients, young and old, I found intubating conditions adequate, vocal cord damage nil, dental damage unremarkable, patient satisfaction excellent, efficiency acceptable, and safety superior. There were no episodes of sudden, unexpected oxygen desaturation, fire, airway obstruction, and aspiration. Respiratory assistance was simple and easy. IV access and fentanyl supplementation to optimize vocal cord relaxation was routinely accomplished after induction, but was not essential. Patients were maintained with $1 / 2$ MAC inhalation agent supplemented with small doses of fentanyl. The nasotracheal tube was left in place to prevent airway obstruction during transport to the recovery room, and was removed by the nursing staff after patients demonstrated purposeful movements. The technique facili- 
tated rapid recovery and discharge.

I trained nurses to perform mask induction and nasal intubation under supervision. A Bullard laryngoscope was successfully employed without paralysis for difficult intubations. Failed intubation was rare, and the only consequence was optimized awake intubation.

Elective Sevoflurane mask induction and intubation without paralysis is nearly as efficient as intravenous induction, and far safer. It offers a comfortable and safe means to assess and avoid anticipated airway problems. Better still, it provides the means to eliminate unanticipated induction disasters if routinely employed.

\section{References}

1. Bermudez, E.A., Chen, M.H. Cardiac arrest associated with intravenous propofol during transesophageal echocardiography before DC cardioversion. (2002) Heart Dis 4(6): 355- 357.

2. Smith, T.E., Elliott W.G. Routine inhaled induction in adults: a safe practice? (2006) Anesth Analg 102(2): 646- 647.

3. van den Berg, A.A., Chitty, D.A., Jones, R.D., et al. Intravenous or inhaled induction of anesthesia in adults? An audit of preoperative patient preferences. (2005) Anesth Analg 100(5): 1422- 1424.

4. Brown, E. Physiological effects of high concentrations of ca. (1930) U S Nav Med Bull.

5. Eisele, J.H., Eger, E.I.2nd., Muallem, M. Narcotic properties of carbon dioxide in the dog. (1967) Anesthesiology 28(5): 856865.

6. Beecher, H.K., Todd, D.P. A study of the deaths associated with anesthesia and surgery: based on a study of 599, 548 anesthesias in ten institutions 1948-1952, inclusive. (1954) Ann Surg 140(1): 2- 35.

7. Benumof, J.L. Management of the difficult adult airway. With special emphasis on awake tracheal intubation. (1991) Anesthesiology 75(6): 1087- 1110.

8. Benumof, J.L. Awake intubations are alive and well. (2015) Can J Anaesth 62(7): 723- 726. 